\title{
Modelling geomagnetically induced currents in midlatitude Central Europe using a thin-sheet approach
}

\author{
Rachel L. Bailey ${ }^{1}$, Thomas S. Halbedl ${ }^{2}$, Ingrid Schattauer ${ }^{3}$, Alexander Römer ${ }^{3}$, Georg Achleitner ${ }^{4}$, \\ Ciaran D. Beggan ${ }^{5}$, Viktor Wesztergom ${ }^{6}$, Ramon Egli $^{1}$, and Roman Leonhardt ${ }^{1}$ \\ ${ }^{1}$ Zentralanstalt für Meteorologie und Geodynamik, Vienna, Austria \\ ${ }^{2}$ Institute of Electrical Power Systems, Graz University of Technology, Graz, Austria \\ ${ }^{3}$ Geologische Bundesanstalt, Vienna, Austria \\ ${ }^{4}$ Austrian Power Grid AG, Vienna, Austria \\ ${ }^{5}$ British Geological Survey, Edinburgh, Scotland \\ ${ }^{6}$ MTA CSFK Geodetic and Geophysical Institute, Sopron, Hungary \\ Correspondence to: Rachel L. Bailey (rachel.bailey@zamg.ac.at)
}

Received: 3 March 2017 - Revised: 12 May 2017 - Accepted: 12 May 2017 - Published: 22 June 2017

\begin{abstract}
Geomagnetically induced currents (GICs) in power systems, which can lead to transformer damage over the short and the long term, are a result of space weather events and geomagnetic variations. For a long time, only high-latitude areas were considered to be at risk from these currents, but recent studies show that considerable GICs also appear in midlatitude and equatorial countries. In this paper, we present initial results from a GIC model using a thinsheet approach with detailed surface and subsurface conductivity models to compute the induced geoelectric field. The results are compared to measurements of direct currents in a transformer neutral and show very good agreement for shortperiod variations such as geomagnetic storms. Long-period signals such as quiet-day diurnal variations are not represented accurately, and we examine the cause of this misfit. The modelling of GICs from regionally varying geoelectric fields is discussed and shown to be an important factor contributing to overall model accuracy. We demonstrate that the Austrian power grid is susceptible to large GICs in the range of tens of amperes, particularly from strong geomagnetic variations in the east-west direction.
\end{abstract}

Keywords. Geomagnetism and paleomagnetism (geomagnetic induction)

\section{Introduction}

In March 1989 a geomagnetic storm caused a $9 \mathrm{~h}$ long blackout in the Québec Hydro power grid (Allen et al., 1989). This outage was the result of large geomagnetically induced currents (GICs) affecting the power grid and transformers within the electrical infrastructure. The Québec blackout is just one extreme example of the dangers of very large GICs, with other direct consequences being for example the October 2003 transformer collapse near Malmö in Sweden (Wik et al., 2008).

GICs are a consequence of clouds of energetic particles from the Sun interacting with the Earth's magnetic field causing geomagnetic storms and rapid geomagnetic variations, which in turn induce geoelectric fields in the Earth's surface (e.g. Boteler and Pirjola, 1998). This leads to the development of electric potentials over large distances. With the arrival of extensive electrical infrastructure, new paths of least resistance for direct current flow have been created, and currents can flow through the power grid or gas pipelines (Lehtinen and Pirjola, 1985; Pirjola et al., 2000). With transformers as the power grid's earthing points, this leads to quasi-direct currents passing through the transformers, which in the long and the short term can cause transformer damage. Direct currents in transformer windings can lead to half-cycle saturation of the transformer at levels of tens of amperes (Molinski, 2002). In mild cases, this can lead to reduced AC transmission capability, transformer heating (Price, 2002; Pirjola, 
2002), and possibly a shorter transformer life (Zois, 2013). In the worst case it can lead to transformer fires and complete transformer failure (Gaunt and Coetzee, 2007; Wik et al., 2008).

It is therefore important to study, model, and predict the possible GICs that could affect a conductive network. Highlatitude countries (in particular those in the auroral zone band of $55-70^{\circ}$ geographic latitude, where the auroral electrojet dominates) and regions of high ground resistivity are most susceptible to GICs (Boteler et al., 1998), and there have been several studies conducted in these areas (Viljanen and Pirjola, 1994; Beamish et al., 2002; Wik et al., 2008; Myllys et al., 2014). Research into GICs in lowlatitude and equatorial countries such as the Czech Republic (Hejda and Bochníček, 2005), Brazil (da Silva Barbosa et al., 2015), Spain (Torta et al., 2012), Greece (Zois, 2013), Japan (Watari et al., 2009), South Africa (Bernhardi et al., 2008; Matandirotya et al., 2015), Australia (Marshall et al., 2011), and New Zealand (Beland and Small, 2005), which were previously considered to be at low risk from all but the most extreme geomagnetic storms, show that considerable GICs (in the range of tens of amperes) do also appear at lower latitudes. In these regions, large geomagnetic variations have been shown to result from ring current intensification, where solar wind is the driving force (Kappenman, 2005).

Austria, being a midlatitude country, is not expected to experience dangerously large GICs; however, due to the presence of the highly resistive alpine rocks stretching across the country, it is more at risk than areas at the same latitude. As can be seen in the study of general European GIC risk in Viljanen et al. (2014) (cf. Fig. 5) based on subsurface conductivity models from Ádám et al. (2012), Austria has a GIC risk comparable to that of areas in the lower northern auroral zone such as Denmark and Scotland.

In 2014, during the commissioning of a new transformer designed for very low sound emissions, unexpected noise was noticed by the Austrian Power Grid (APG), and saturation due to DC currents or very low-frequency currents (below $1 \mathrm{~Hz}$ ) was assumed to be the reason. The higher levels of noise were eventually correlated with geomagnetic activity. Soon after, in partnership with the APG, studies into the presence and nature of GICs in Austria were started (Halbedl et al., 2014, 2016).

In this paper we will describe the usage of a thin-sheet approximation to model geoelectric fields in Austria. Both surface and subsurface ground conductivities are taken into account when modelling the induced geoelectric field from geomagnetic field variations, and these are detailed in Sect. 2 along with the model. Using the approach originally described by Lehtinen and Pirjola (1985), we then show in Sect. 3 how modelled direct currents resulting from the geoelectric potentials flow through the power grid network. We will present results from the model compared to direct measurements of GICs at a transformer neutral point. The accu- racy and applicability of the results will be summarised and discussed in Sect. 4.

\section{Modelling GICs}

The modelling of GICs is achieved through two steps: the first step, often labelled the geophysical part, is the modelling of the electric field induced in the resistive, layered ground by geomagnetic variations. The second step, or engineering part, is the calculation of the flow of currents through a power grid with grounding points across the Earth's surface. Here, we present all aspects and principles applied in the modelling of GICs in the Austrian power grid.

\subsection{Thin-sheet model}

For the first step, calculations of the distribution of the geoelectric field from the geomagnetic variations are done using a thin-sheet model. This model was originally developed by Vasseur and Weidelt (1977) based on concepts described in Price (1949) to model induction anomalies. The model is used to solve quasi-3-D induction problems in areas with large lateral conductivity variations near the surface. In the model, a 2-D thin-sheet of varying lateral conductivity sits atop a 1-D half-space of conductivity layers going into the Earth, and therefore more complex surface currents resulting from shallow conductivity variations can be accounted for without requiring a full 3-D representation. The version used in this study is based on code which has been used in modelling GICs in the UK in the past (Thomson et al., 2005).

The model input variables are the geomagnetic variations and the surface and subsurface conductivity models. The geomagnetic variations are passed into the calculations in the form $\Delta B_{x} / \Delta t$ and $\Delta B_{y} / \Delta t$, where $\Delta B_{i}$ is the change in the $i$ component of the geomagnetic field over a certain time period $\Delta t$. This means that the analysis occurs primarily in the time domain. The length of the period that can be used is constrained by requirements that must be fulfilled for the model to be physically viable. These requirements are touched on by Weaver (1982) and summarised by Heinson and Lilley (1993). They primarily describe relations between the relative skin depths of the thin sheet and the underlying medium. The skin depth is given by

$\delta=\sqrt{\frac{2 \rho}{\omega \mu}}=\sqrt{\frac{\rho}{\pi f \mu}}$.

It is dependent on both the frequency or period and the resistivity of the given medium. After applying the constraints to all available subsurface conductivity models in Austria, we find that the available periods to allow comparison between models must be greater than $40 \mathrm{~s}$ and less than $400 \mathrm{~s}$. To save on computation time, we choose a value of $\Delta t=300 \mathrm{~s}$ that 
will be used for all results throughout this paper. The validity of this period for modelling geomagnetic storm signals is examined in the discussion.

Measurements of the geomagnetic variations are taken from the Conrad Observatory and used to calculate the variation in the field per period (here $300 \mathrm{~s}$ ) in nanotesla per period. These variations are assumed to be the same across Austria. Although there are other methods available to model the changing geomagnetic variations across regions (e.g. spherical elementary current systems as described in Amm and Viljanen, 1999), the south of Austria is largely unconstrained due to a general lack of geomagnetic stations and useful data, which is a potential source of inaccuracies. This will hopefully be expanded on in the future with the set-up of a new geomagnetic station in the south of Austria. In the meantime, Austria's relatively small size means that the assumption of equivalent geomagnetic variations across its area is reasonable and is not expected to significantly affect the results.

The thin-sheet model described above takes geomagnetic variations and models of the ground conductivity to compute the geoelectric field strength as a function of position in Austria. To compute the field as it changes over time, the day is broken down into 288 windows with $\Delta t=300 \mathrm{~s}$, each of which is computed individually.

For the second step, the flow of GICs in the network as a result of the geoelectric field is calculated for each window using the Lehtinen-Pirjola method described in Lehtinen and Pirjola (1985), which treats the power grid as an electric circuit and uses Ohm's and Kirchoff's laws to determine the flow of current.

For this to be valid, we must assume that the system is primarily time-independent; however, the induced fields vary so slowly with time (usually in the range of millihertz) that they can be considered constant. This allows us to ignore contributions to the magnetic field caused by a varying electric field.

The calculation of GICs using the Lehtinen-Pirjola method reduces to the following equation in matrix form:

$\mathbf{I}=\left(\mathbf{1}+\mathbf{Y}^{n} \mathbf{Z}\right)^{-1} \mathbf{J}$

where $\mathbf{I}$ is the GIC at all grid nodes or transformer earthing points and $\mathbf{1}$ is the identity matrix. The value of $\mathbf{I}$ relies on the following variables: the earthing impedance $\mathbf{Z}$, which is taken to be only the earthing resistance at each node due to the assumed time independence of the potentials and the fact that no two stations are close enough to influence each other's potential; the network admittance matrix $\mathbf{Y}^{n}$; and the "perfect earthing" or grounding currents $\mathbf{J}$, which are dependent on the potential difference between two nodes and the resistance of the line connecting them. The resulting current I at each node can be positive or negative, representing currents flowing into or out of the Earth, respectively.

\subsection{Conductivity models of Austria}

The thin-sheet approach described above models the geoelectric field induced by a changing external magnetic field in an earth with resistivity layers varying with depth. For a full geoelectric field model, we combine two different types of conductivity models: firstly, lateral conductivity variations are taken into account with a 2-D model of the ground conductivity at the surface of Austria, and secondly, varying subsurface resistivity layers are represented by a 1-D half-space model of the subsurface resistivity as a function of depth into the Earth.

In order to develop a detailed conductivity model of Austria, the approach of Beamish (2012) was followed, in which electromagnetic data, available from more than 50 aerogeophysical campaigns carried out between 1980 and 2014 by the Geological Survey of Austria (GBA), were partly reprocessed with modern techniques. The results are ground conductivity (or resistivity) data sets of homogeneous halfspace inversion results as well as conductivity multilayer information of the survey areas. In the next step, these data sets were correlated with a hydrogeological map of Austria on a scale of $1: 500000$ and average conductivity values were derived for each of the hydrogeological units. Additionally, information on ground conductivity values from more than 1000 multi-electrode geoelectric profiles was available to supplement the subsurface information to complete the entire Austrian territory. Using available geological maps for outside of Austria, the conductivity was extrapolated to a rectangular region resulting in a high-resolution subsurface conductivity map of Austria and its surroundings, which is shown in Fig. 1.

The inversion program EM1DFM (Version 1.0, University of British Columbia, 2000) which was used constructs onedimensional models of conductivity at each measurement point. For a layered model it is necessary to predefine the depth of each interface. For the task required in this work, a homogeneous half-space is assumed. Interpolating the models of each sounding results in a conductivity distribution of around 50 to $100 \mathrm{~m}$ thick subsurface layers inside the survey areas. The variation in volume of the captured layer depends on the conductivity of the subsurface and the height of the measurement system above the ground. All soundings were conducted from a height of roughly $50 \mathrm{~m}$ with transmitting coils generating an electromagnetic field with the frequencies $340,3200,7190$, and $28850 \mathrm{~Hz}$ and a sampling rate of 10 measurements per second (resulting in a $3 \mathrm{~m}$ measuring point distance). Using these frequencies, the depth of conductivity values that can be determined from the results depends on the local ground conductivity. At $3200 \mathrm{~Hz}$, the skin depth in areas with ground conductivity of 200 to $2 \mathrm{mS} \mathrm{m}^{-1}$ ranges from roughly 14.1 to $140.7 \mathrm{~m}$. At the highest frequency of $28850 \mathrm{~Hz}$, the skin depth ranges from 4.7 to $46.8 \mathrm{~m}$.

The hydrogeological map of Austria (Schubert, 2003) on the scale of $1: 500000$ is suitable for the purpose of general- 


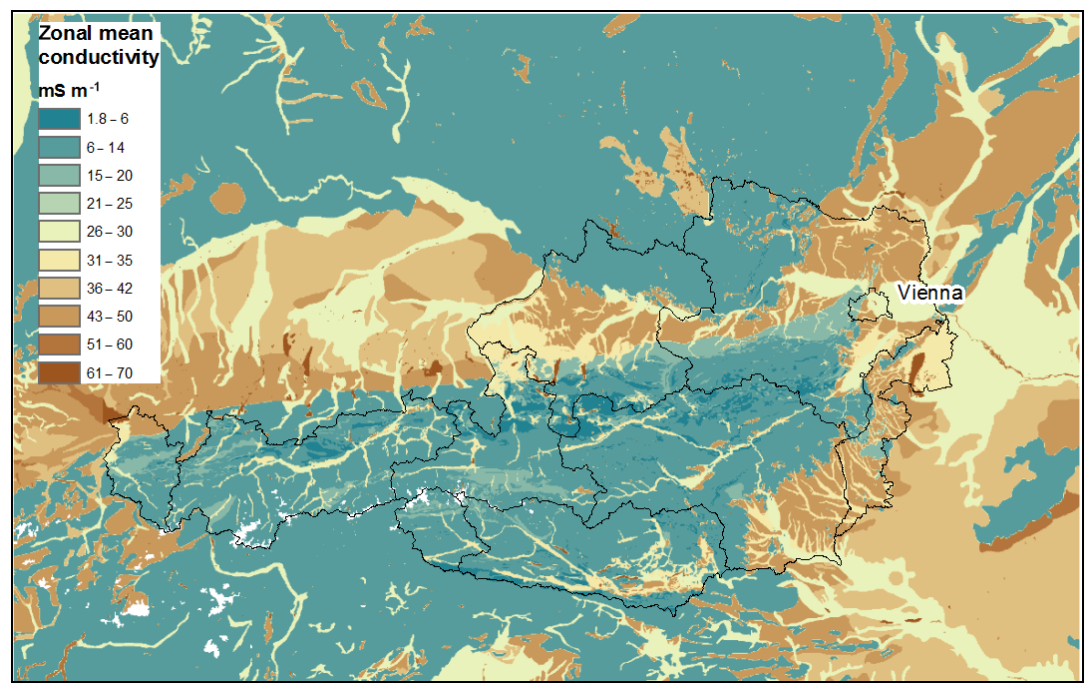

Figure 1. High-resolution subsurface conductivity model of Austria and its surroundings produced by the GBA.

ising airborne electromagnetic data because hydrogeological parameters such as grain size and water content are determining factors that influence the range of measured conductivities. This map considers both hydrological and lithological aspects. For the areas surrounding Austria, the international hydrogeological map of Europe (1:1500 000; Duscher and Günther, 2014) was used.

The 1-D layer conductivity model was taken from the European RHO Model (EURHOM) developed by Ádám et al. (2012), which was derived from a collection of magnetotelluric data sets spanning most of Europe. In this model, areas of certain resistivities are represented by rectangular cells. The area of Austria and its borders is covered by a total of six different cells as shown in Fig. 2. A table showing the four main model resistivities and associated depths can be found in Table 1 (models 15 and 17 were left out as they were not very relevant to the power grid and model). As can be seen in the table, each layer is described by the vertical size of the layer $\left(d_{m}\right)$ and a value of resistivity $\left(\rho_{m}\right)$. These values range from hardly resistive $(5 \Omega \mathrm{m})$ to highly resistive $(10000 \Omega \mathrm{m})$ and span total depths ranging from 60 to $152 \mathrm{~km}$. The bottom value for each cell is assumed in EURHOM to remain the same indefinitely with growing depth. In application we applied the condition of $\rho \rightarrow 0$ over depths of many hundreds of kilometres with an exponentially decaying resistivity beyond a maximum depth of $200 \mathrm{~km}$.

\subsection{The Austrian power grid}

The APG is the transmission system operator in Austria and is also responsible for the control area of Austria. The APG grid consists of 110,220 , and $380 \mathrm{kV}$ lines and substations. The overall system length of the transmission grid is over $6700 \mathrm{~km}$, with a total of 60 substations operating in Austria. For the simulation model, only the 220 and $380 \mathrm{kV}$ volt-

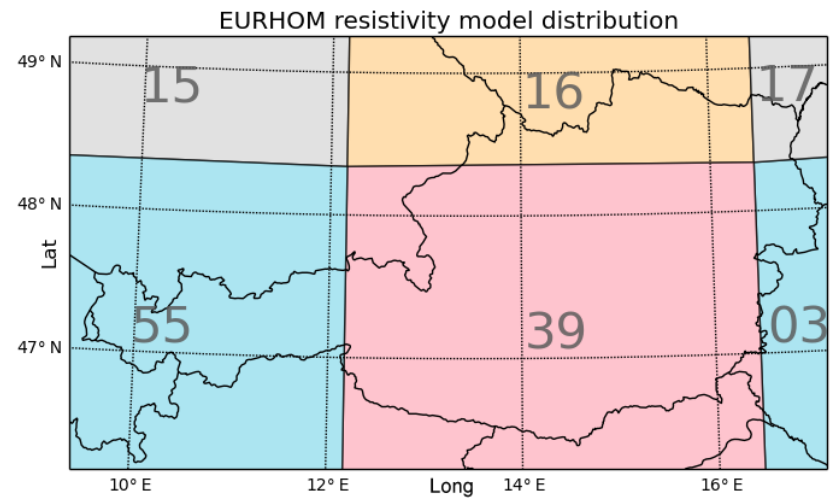

Figure 2. Cells of EURHOM layer resistivity models across Austria.

age levels are considered and lower levels are ignored due to more highly resistant lines and different neutral point treatments of transformers (such as connection to earth via an inductor or being generally isolated), making them less relevant for GICs. If the distance between two neighbouring nodes is less than $7 \mathrm{~km}$, they are merged. The power grid used in the model is a combination of the APG grid in Austria and the nearest stations in the surrounding countries (Germany, the Czech Republic, Slovakia, Hungary, Slovenia, Italy, and Switzerland). There are a total of 113 station nodes in the model network, 43 of which are within Austria and managed by the APG. The lines are comprised of both 220 and $380 \mathrm{kV}$ lines, and transformer winding resistances of $0.06 \Omega$ per phase and $0.2 \Omega$ per phase, respectively, are assumed across the grid. These resistance values were taken as standard values from literature (cf. Horton et al., 2012). In addition, it is assumed that only one transformer on the 380 and $220 \mathrm{kV}$ level per substation is grounded. The resistance of the 
Table 1. EURHOM resistivity models from Ádám et al. (2012) covering most of Austria.

\begin{tabular}{rr|rr|rr|rr}
\hline \multicolumn{2}{c|}{03} & \multicolumn{2}{c|}{16} & \multicolumn{2}{c|}{39} & \multicolumn{2}{c}{55} \\
\hline$d(\mathrm{~km})$ & $\rho(\Omega \mathrm{m})$ & $d(\mathrm{~km})$ & $\rho(\Omega \mathrm{m})$ & $d(\mathrm{~km})$ & $\rho(\Omega \mathrm{m})$ & $d(\mathrm{~km})$ & $\rho(\Omega \mathrm{m})$ \\
\hline 3.00 & 5.00 & 20.00 & 1500.00 & 55.00 & 1000.00 & 0.90 & 110.00 \\
57.00 & 1000.00 & 20.00 & 500.00 & 45.00 & 300.00 & 1.10 & 30.00 \\
$\infty$ & 10 & 110.00 & 800.00 & $\infty$ & 1000.00 & 150.00 & 10000.00 \\
& & $\infty$ & 10.00 & & & $\infty$ & 90.00 \\
\hline
\end{tabular}
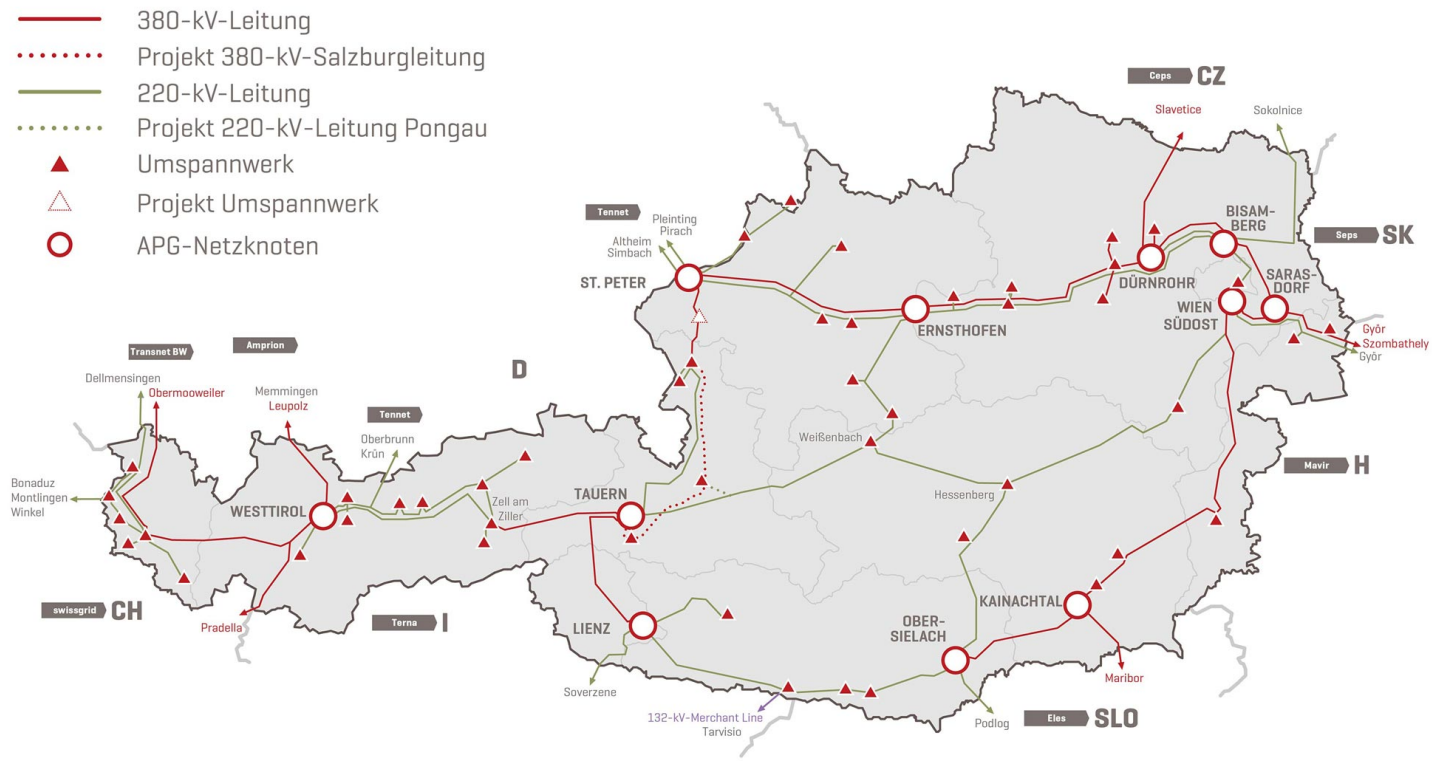

Figure 3. Depiction of the Austrian power grid provided by the APG. Triangles represent substations while circles represent network nodes. Red and grey lines are 380 and $220 \mathrm{kV}$ lines, respectively.

grounding is set to $0.2 \Omega$ at all substations. A representation of the grid can be seen in Fig. 3.

When modelling the DC equivalent of power transformers in the single-phase network admittance matrix, the different galvanic connections from the voltage levels to the ground and between the windings have to be considered (Boteler and Pirjola, 2014). For a step-up transformer, the high-voltage level is connected through the winding resistor to the grounding resistance of the substation. In the case of a two-winding transformer, there are windings for each voltage level connected to the neutral bus. The neutral bus is a node between the windings and the grounding resistance of the substation. A two-winding autotransformer DC model differs from those mentioned above. The series winding connects the high-voltage with the low-voltage level but not with the neutral bus. With the common winding, a path between the low-voltage level and the neutral node occurs. The grounding resistance of the substation connects the neutral bus to earth.

\section{Results}

The GIC model parameters described above were applied and the resulting GIC values have been compared to quasiDC currents measured directly at a transformer in the south of Vienna. The Conrad Observatory is also south of Vienna at a distance of around $50 \mathrm{~km}$ from the measurement location, meaning the input magnetic field is representative of the field at the transformer site.

Generally, it is quite challenging to monitor low direct currents in the presence of high alternating currents, and the current measurement device has gone through iterations for improvement over time. Direct currents at the transformer neutral point have been measured using a current clamp with Hall sensor technology. The signal from the current probe is filtered by a passive second-order low-pass filter with a cutoff frequency of $1 \mathrm{~Hz}$, thus keeping the very low-frequency components and removing the rest. Afterwards the signal is recorded by a data logger. Initially there was an upper limit on the measured values of $\pm 2.3 \mathrm{~A}$ (with a sample interval every $60 \mathrm{~s}$ ), as the currents were not expected to exceed this 


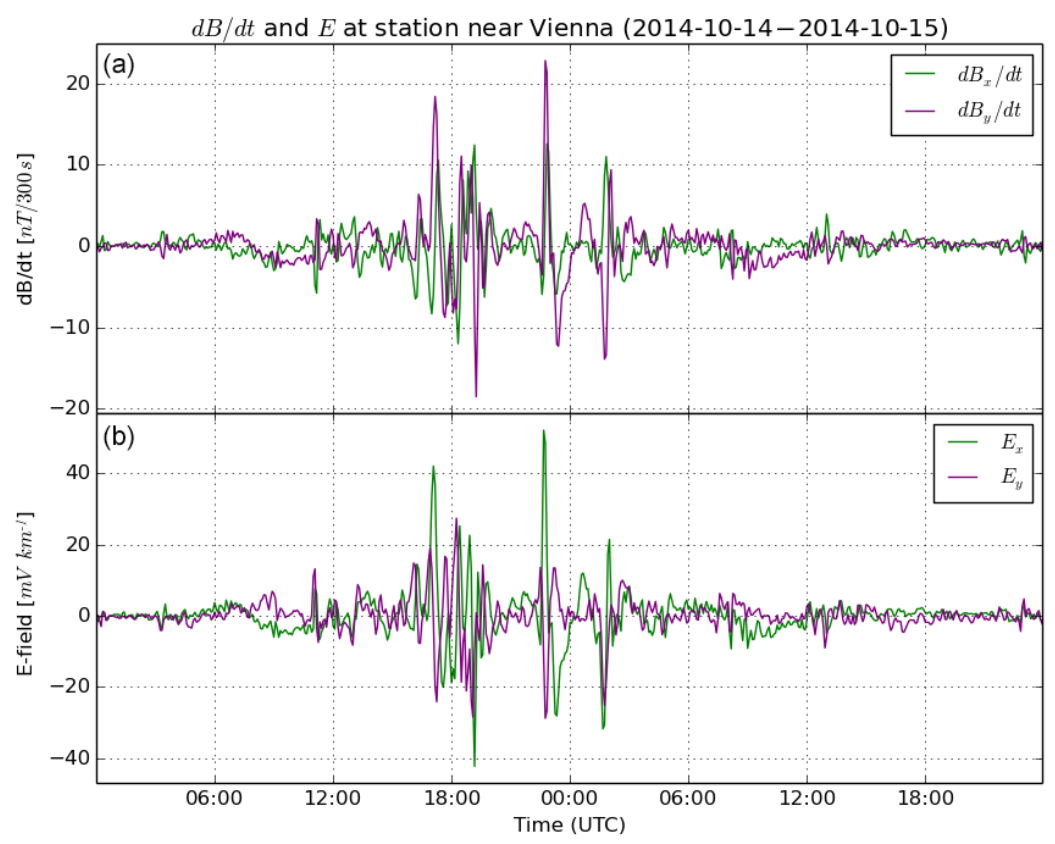

Figure 4. (a) Change in the horizontal geomagnetic field at the Conrad Observatory ( $x$ and $y$ components) per period of $300 \mathrm{~s}$ for the days of 14 and 15 October 2014. Change in the $x$ direction is in green; change in the $y$ direction is in purple. (b) Modelled geoelectric field for the area near Vienna on the days of 14 and 15 October 2014. The field in the north-south direction (Ex) is plotted in green; the east-west direction (Ey) is plotted in purple.

value. This figure was exceeded multiple times during the measurements, and we cannot know what the actual value would have been. The measurement device has since been updated to account for greater currents and will allow for more detailed studies in the future.

The measurements took place in intermittent periods between August 2014 and August 2015, with a total of 147 days of clean and unbroken $24 \mathrm{~h}$ measurements. Despite the measurements taking place during a relatively weak solar maximum period, there were few examples of geomagnetic storms and the associated rapid geomagnetic variations to draw conclusions on the amplitudes of extreme events. As such, we have chosen one example of a weaker geomagnetic storm (spanning the days 14 and 15 October 2014) to illustrate the model and results.

Figure 4 shows the geoelectric field values computed by the thin-sheet model using geomagnetic variations $(\mathrm{d} B / \mathrm{d} t)$ at the Conrad Observatory and the surface and subsurface conductivity models as input. Here, we can see that the maximum field strength reached is $52 \mathrm{mV} \mathrm{km}^{-1}$ in the $E_{x}$ component. This corresponds to a $\mathrm{d} B / \mathrm{d} t$ value of $23 \mathrm{nT} / 300 \mathrm{~s}$ as also seen in Fig. 4.

The GICs that are computed from the modelled geoelectric field using the Lehtinen-Pirjola method are shown in Fig. 5. As can be seen from this example, most modelled GIC behaviour matches the measured DC in both shape and magnitude reasonably well. The coefficient in the top left, $r$ for the Pearson's correlation coefficient, shows quantita- tively that there is also a good match in form. The maximum amount of GICs observed here was $2.0 \mathrm{~A}$.

Note that during the measurements shown in Fig. 5, the measuring device was saturated at the following times: 14 October 2016 17:00:00 UTC, 14 October 18:23:00, 14 October 2014 22:45:00, and 15 October 2016 01:48:00. We cannot draw any conclusions on the exact size of the currents flowing through the transformer during these times other than to say that they were larger than $\pm 2.3 \mathrm{~A}$ (currents in the figure larger than this are due to a level offset). As there were unknown scales of currents present, this could mean that the model is underestimating the GICs. One such saturated period lasted $15 \mathrm{~min}$, suggesting that the currents were much larger during this time.

A further observation is that there is a long-period (several hours) signal mid-morning on both days that is only barely accounted for in the model. We will call this the diurnal signal. As the measurement device was kept at a roughly constant temperature throughout measurement periods, it is unlikely that this is a result of daily temperature variations, and it is more likely a result of the solar-quiet daily variation (Sq) in the horizontal component geomagnetic field (see, e.g., Matsushita and Maeda, 1965). This variation is caused by the influence of the Sun on the sunlit part of the ionosphere and the resulting current systems and is greatest during the daytime hours.

The diurnal signal can be quantified independently of other variations by averaging the daily variations in both the mea- 


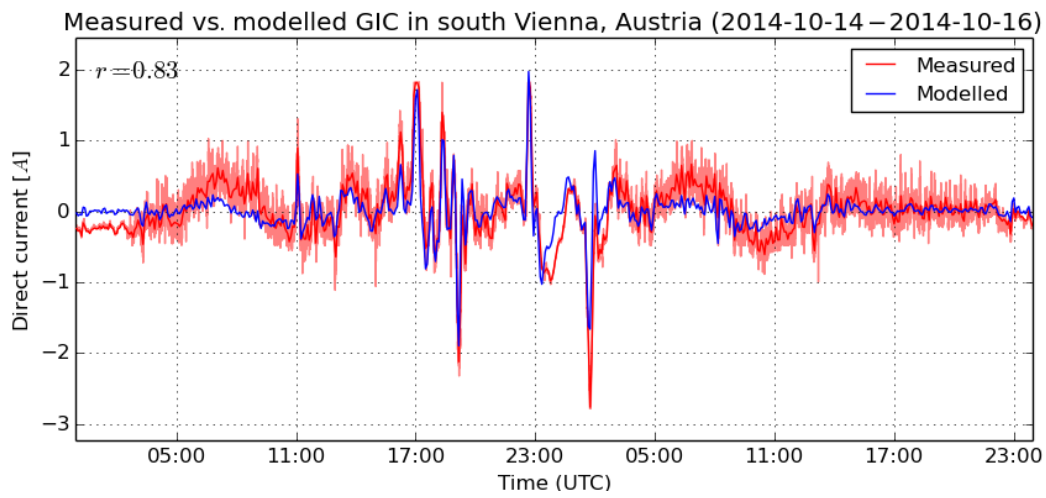

Figure 5. Modelled GICs (blue) in comparison to the measurements (red). The raw measurements are plotted in lighter red and the solid red line are the measurements that have been filtered to remove noise and reduce to the same sampling rate of $300 \mathrm{~s}$ for comparison to the model. The value in the top left is the Pearson's correlation coefficient $r$ between the model and filtered measurements.

sured DC and modelled GICs over a span of days. The longest available unbroken period of measurements was the whole of the month of October in 2014 with a few additional days for a total of 34 averaged days. It is important in this case to restrict the analysis to one time of year due to the seasonal variation in the $\mathrm{Sq}$ variation in the geomagnetic field. Plotted in Fig. 6 is the result of measurements and model outcomes being averaged over all 34 days as well as the residuals. Plotted alongside the averages for comparison is the mean-subtracted geomagnetic field variation in the horizontal component $H$ for the same period. The DC measurements have been filtered to remove a prevalent and regularly recurring $15 \mathrm{~min}$ signal, which originates from the power system itself. As can be seen clearly in the figure, both the modelled and measured GIC variations contain a long-period signal between the times 05:00 and 14:00 UTC, but they appear to be phase-shifted. Other shorter-period signals that appear later in the day (later than 14:00) are not phase-shifted.

We can quantify the amount of phase shift by looking at the cross-correlation between the model and measurements, shown on the right side of Fig. 6. The upper plot shows the cross-correlation between averaged measurements and model results during the diurnal variation time, which should be 0 but is offset by 10 data points (maximum shown in red). In our case of $\Delta t=300 \mathrm{~s}$, this equates to a time lag of the model behind the measurements of $\tau=3000 \mathrm{~s}$ or $50 \mathrm{~min}$. A sine fit to both signals in the restricted time period results in fitted periods of $P_{\mathrm{DC}}=2.96 \times 10^{4} \mathrm{~s}$ and $P_{\text {model }}=2.82 \times 10^{4} \mathrm{~s}$ $(8 \mathrm{~h})$. The equivalent phase shift between the two using $P_{\mathrm{DC}}$ is $\varphi=36.4^{\circ}$, which is considerable and unexpected.

The maximum cross-correlation for the rest of the day (Fig. 6, lower right), in contrast, behaves as expected and shows no lag, as do all analyses of individual days; therefore, we can exclude the possibility of this effect being caused by an artefact in the model. We will investigate the cause of this localised phase shift in the discussion.

\subsection{Susceptibility of grid to field orientation}

Following the approach used in Pirjola (2008), Torta et al. (2014), and most recently Blake et al. (2016), a uniform horizontal geoelectric field of $1 \mathrm{~V} \mathrm{~km}^{-1}$ in all possible field orientations is applied to the grid to evaluate the susceptibility of particular substations and sections in the grid to varying field directions. A field strength of $1 \mathrm{~V} \mathrm{~km}^{-1}$ would only arise as a result of particularly strong geomagnetic variations, and the results are also a useful indication of the GICs that can be expected during extreme geomagnetic storms.

The results of this analysis are plotted in Fig. 7. Each circle represents a node, with the size of the circle the magnitude of the GICs experienced at that node. At each station, the maximum GICs resulting from an electric field of $1 \mathrm{~V} \mathrm{~km}^{-1}$ in all possible directions has been plotted and thereby reveals which stations are at risk of particularly large currents. The red arrows show the direction of the electric field leading to the maximum levels of GICs $\left(0^{\circ}=\mathrm{N}-\mathrm{S}, 90^{\circ}=\mathrm{E}-\mathrm{W}\right.$, etc. $)$. Only the maxima are plotted here, although a reversed field (rotated by $180^{\circ}$ ) would result in the same amount of current flowing in the opposite direction. The largest resulting current in the stations controlled by the APG is $49.13 \mathrm{~A}$ in southern Vienna for a $\mathrm{N}-\mathrm{S}$-oriented electric field. In contrast, the maximum GICs seen with an applied E-W-oriented electric field is only $82 \%$ of this value at $40.21 \mathrm{~A}$. The largest value in the whole network (including surrounding countries) was 49.38 A in western Slovakia for an electric field in the NWSE direction.

In summary, we see that the Austrian power grid is more susceptible to northerly geoelectric fields or strong east-west geomagnetic field variations, such as can be seen during substorm periods in geomagnetic storms.

\subsection{Spatial distribution of GICs in Austria}

As can be seen in Fig. 7, the largest currents are seen primarily in the east and west of Austria (near Vienna and Inns- 

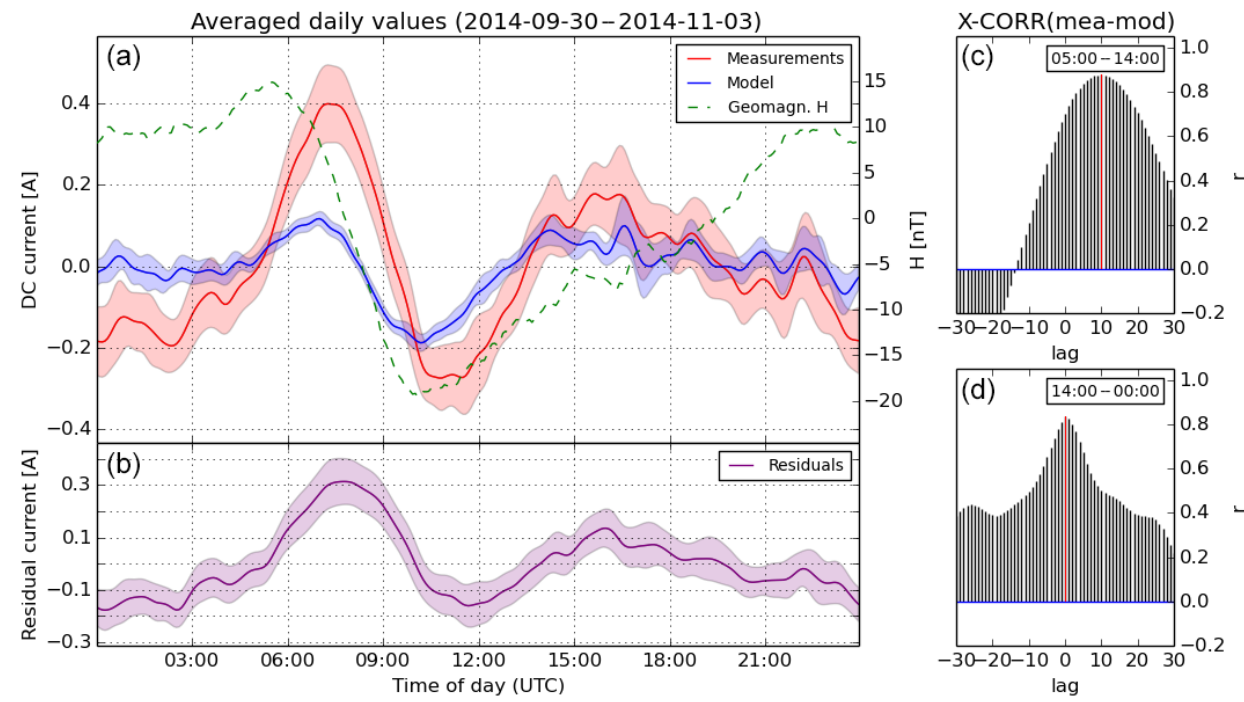

Figure 6. Panel (a) displays the averaged daily variations in measured DC currents (red) and the model results (blue) with $95 \%$ confidence intervals, while (b) shows the averaged residuals between the measurements and models, revealing signals that are not covered by the model. (c, d) The cross-correlation between model and measurements within the diurnal signal (c) and outside (d), with the maxima highlighted in red.

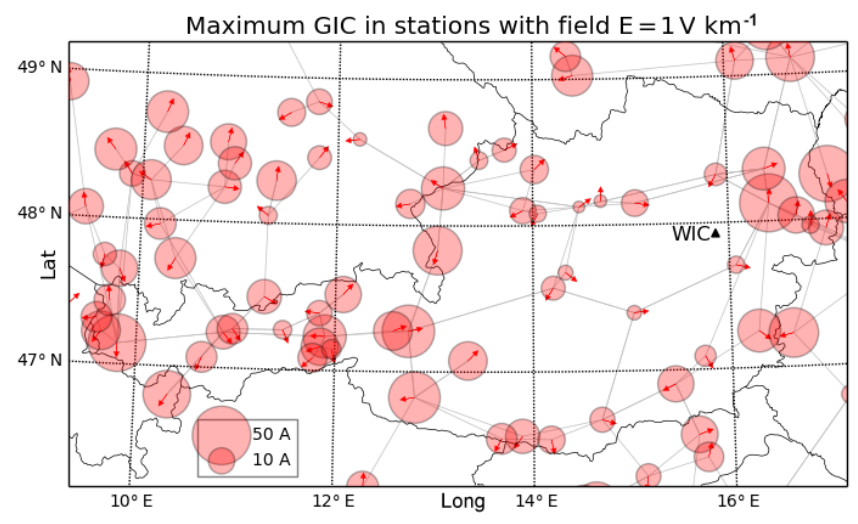

Figure 7. Maximum GICs at the various stations in the Austrian and nearby network for all orientations of a geoelectric field with magnitude $1 \mathrm{~V} \mathrm{~km}^{-1}$. Each circle represents a node, and the line connections between nodes are shown in grey. The size of the circle represents the magnitude of GICs, while the arrows depict the geoelectric field orientation that leads to a maximum at that node. The position of the Conrad Observatory (WIC) is marked by the black triangle.

bruck) with some central spots also seeing larger GICs. As the Austrian power grid is connected to those of its neighbours, there are no edge affects as seen in countries with, e.g., coastlines where the grid has a defined edge, but considerable GICs can still arise. Generally, nodes sitting on north/northwest-running lines experience the largest amounts of GICs, roughly twice as much as nodes sitting only on eastrunning lines.

\section{Discussion}

Analyses of geomagnetically active days in the data such as the period in October presented in the figures show very good correlation $(r>0.8)$ between the model and measurements, which is what we would hope for in order to correctly model large and potentially dangerous currents that would only appear during geomagnetically active storm times. This good correlation does not, however, carry over to geomagnetically quiet days, where the model fit is not optimal $(r \sim 0.5-0.7)$. The reason for this is partly due to non-geomagnetically induced current signals prevailing at lower-magnitude DC but is also largely a result of the dominance of the "diurnal signal" noted above, and it becomes clear that the model does not accurately represent the long-period signal seen in the measurements. Therefore, we wish to evaluate the source of this signal in order to discover areas in which the model is lacking and to better model all types of signals.

\subsection{Impedance phase shift}

We can first approach this as a quasi-static system in which the source of the phase shift is the components of the power grid itself. The circuit formed by the ground potential between two nodes and the lines that join them can be treated as an RLC circuit or resonant circuit. Here, we review the concept of impedance phase, which is the phase shift by which an alternating current lags the voltage. We will treat the diurnal signal as a very slowly varying $\mathrm{AC}$ current and the circuit as one in which the ground potential is the applied voltage and the transformer earthing resistances and the line resistances in series make up the circuit. The phase shift between 
the voltage and current in a circuit with resistors, capacitors, and inductors can be calculated using the following equation:

$\theta=\operatorname{atan}\left(\frac{X_{L}-X_{C}}{R}\right)$,

where the variables in the numerator are the inductive and capacitive reactances, respectively, calculated using the formulae $X_{L}=2 \pi f L$ and $X_{C}=1 /(2 \pi f C)$. In a purely resistive circuit with $X_{L}-X_{C}=0$, the phase shift would be $\theta=0^{\circ}$.

In the simple case of a homogeneous electric field, $V \propto$ $E \propto \mathrm{d} B / \mathrm{d} t$ and we can use the geomagnetic variations as proxy for the voltage to calculate the phase shift. A crosscorrelation analysis between the diurnal signal in the measurements and $\mathrm{d} B / \mathrm{d} t$ shows that the current leads the voltage by around $50 \mathrm{~min}\left(\theta_{\text {mea }}=35.8^{\circ}\right)$ and the model leads by around $100 \min \left(\theta_{\bmod }=71.6^{\circ}\right)$. There is a residual phase shift of $35.8^{\circ}$, with the measurements lagging the model prediction. If we assume that the grid causes the residual phase shift, then from Eq. (3) we know that it is an inductive circuit. However, in application, a circuit leading to the observed phase shift (with a total resistance of $8 \Omega$ from the transformers and line) would have an inductance of $L=27710 \mathrm{H}$, a highly unrealistic inductance for a circuit. For comparison, a rectangular coil the size of Austria $(\sim 600 \times 300 \mathrm{~km})$ with a wire diameter of $1 \mathrm{~m}$ and a core of iron does not even reach the above value with an inductance of only $\sim 23100 \mathrm{H}$. Although there is likely to be a small phase shift in GICs through a power grid caused by inductive and capacitive components, it would not be to the extent seen here. We can exclude the quasi-static approach and move on to a geophysical interpretation.

\subsection{Phase shift between model and measurements}

It is known in the field of magnetotellurics that there is a phase shift in a geoelectric field induced in a medium that depends on the conductivity of the medium and the analysed frequencies (Wait, 1962), with some showing a decrease with decreasing frequency (e.g. Weckmann et al., 2005). Different signal frequencies travel preferentially inside different layers within their penetration depth, and a phase shift between the measurements and the model could be a result of modelling with an inaccurate subsurface conductivity model or of not reaching an adequate depth while modelling.

As we are only analysing windows of $300 \mathrm{~s}$ in sequence, it is likely that signals with greater periods are not accurately modelled. However, an analysis of the same data in greater windows $(900 \mathrm{~s})$ results in the same shift. Here, we include a power spectrum analysis of the GIC measurements for clarification. Analyses of GICs during geomagnetically active and stormy times, which are our primary interest, show that the dominant signal periods are around $1700 \mathrm{~s}$ or $6 \times 10^{-4} \mathrm{~Hz}$, which is well below the Nyquist frequency of the window used $\left(v_{n}=1.6 \times 10^{-3} \mathrm{~Hz}\right)$ and will be covered by the modelling approach. A power spectrum of the DC measurements

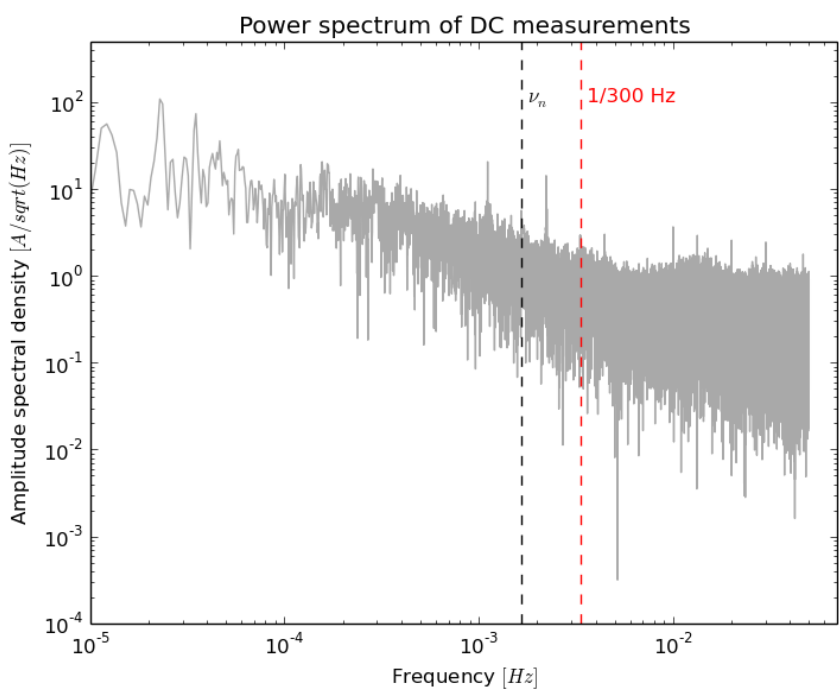

Figure 8. Power spectrum of DC measurements. The window used in our analysis as well as the associated Nyquist frequency of that window $\left(v_{n}\right)$ are displayed as red and black lines, respectively. The two defined spikes in the spectrum around $10^{-3} \mathrm{~Hz}$ result from periodic 15 and $7.5 \mathrm{~min}$ signals related to the power system.

in Fig. 8 shows that signals with frequencies greater than $6 \times 10^{-3} \mathrm{~Hz}(<170 \mathrm{~s})$ are swallowed by white noise.

The only clue as to the actual cause comes from a comparison between a model using the geoelectric field derived from one subsurface layer model and a model using multiple subsurface models. In the simplest case, the GICs will be calculated from an Austria-wide geoelectric field modelled from geomagnetic variations on a single subsurface resistivity layer model (e.g. EURHOM 39). In a more complex and geophysically realistic case, the code extracts geoelectric fields from multiple EURHOMs (four in total; see again Fig. 2) depending on the area of network being modelled. The results from the more complex model using multiple EURHOM cells prove to be better fits to the measurements (an improvement in $r$ averaging 0.05). When put through the same procedure as above in determining the averaged daily variations, the phase shift between the more complex model and measurements is $35 \mathrm{~min}$ instead of the original $50 \mathrm{~min}$. A further test on data from April 2015 with a shorter period of 11 averaged consecutive days, which also shows a phase shift between model and data of $50 \mathrm{~min}$, results in a similar improvement of 50 to $40 \mathrm{~min}$ in time lag. While only a small improvement, this highlights the importance of the lateral geoelectric field variations input parameter in the model, which can be developed further in future model iterations. 


\section{Conclusions}

The thin-sheet approach of geoelectric field modelling has been successfully applied to Austria in order to model the flow of GICs through the national power grid. The model took geomagnetic variations from the Conrad Observatory along with a 2-D thin-sheet surface conductivity model of Austria provided by the GBA and EURHOM 1-D subsurface conductivity models as input. Initial results show good matches with direct DC measurements at a transformer; however, a cut-off level of $\pm 2.3 \mathrm{~A}$ in the measurements limits a more exact analysis of the model and large GICs. There are also signals in the DC measurements that are not captured accurately in the model. These were analysed and linked to the modelling of the field with respect to the subsurface conductivity. A model that includes fields induced by varying subsurface conductivities shows better correlation with the actual measurements. The match between the measurements and model will be the basis for building a more accurate model.

Extrapolating from the model, we see that large GICs are likely to occur primarily in the east and west of Austria, and the grid is most susceptible to large currents caused by eastwest geomagnetic field variations. Direct current measurements at transformer neutrals continue in Austria and are being expanded, which will allow for more detailed analyses and model comparisons in the future.

Code and data availability. The code is available on request from the corresponding author. Data from the Conrad Observatory are available from INTERMAGNET, e.g. at http://www.intermagnet. org/data-donnee/download-eng.php.

Author contributions. RLB conducted the majority of research described in this paper and also drafted the article text. TSH handled the DC measurements at specific points in the Austrian Power Grid and provided these data as well as the power network parameters used in this model. He also wrote parts of the text related specifically to the grid and DC measurements. IS and AR developed the 2-D conductivity model for the surface of Austria from aerogeophysical measurements and provided text for specifications on the model as well as a map plot of said model. GA is an Austrian Power Grid employee and contact for discussions on results, and he also provided text on the power grid for this article. CDB provided the basic model for the GIC analysis and offered discussion input and paper revisions. VW provided data on the subsurface resistivity layers. RE brought further knowledge of power networks and magnetotellurics to the data analysis and revised this paper. RL conceived the study and provided Conrad Observatory data as well as project support and direction. He revised this paper throughout its conception. All authors read and revised the paper before submission.
Competing interests. The authors declare that they have no conflict of interest.

Acknowledgements. The work described here was supported by the FFG in Austria in the project GEOMAGICA (no. 847986). The Austrian Power Grid, the TU Graz, and the Geologische Bundesanstalt are thanked for their data and support, as are the British $\mathrm{Ge}$ ological Survey and the Geodetic and Geophysical Institute.

The topical editor, G. Balasis, thanks J. M. Torta and one anonymous referee for help in evaluating this paper.

\section{References}

Ádám, A., Prácser, E., and Wesztergom, V.: Estimation of the electric resistivity distribution (EURHOM) in the European lithosphere in the frame of the EURISGIC WP2 project, Acta Geodaetica et Geophysica Hungarica, 47, 377-387, https://doi.org/10.1556/AGeod.47.2012.4.1, 2012.

Allen, J., Frank, L., Sauer, H., and Reiff, P.: Effects of the March 1989 solar activity, EOS T., 70, 1479, https://doi.org/10.1029/89EO00409, 1989.

Amm, O. and Viljanen, A.: Ionospheric disturbance magnetic field continuation from the ground to the ionosphere using spherical elementary current systems, Earth Planets Space, 51, 431-440, 1999.

Beamish, D.: The 1: $625 \mathrm{k}$ near-surface bedrock electrical conductivity map of the UK, NERC Open Research Archive, Nottingham, 2012.

Beamish, D., Clark, T. D. G., Clarke, E., and Thomson, A. W. P.: Geomagnetically induced currents in the UK: geomagnetic variations and surface electric fields, J. Atmos. Sol.-Terr. Phy., 64, 1779-1792, 2002.

Beland, J. and Small, K.: Space weather effects on power transmission systems: The cases of Hydro-Québec and Transpower New Zealand Ltd., in: Effects of space weather on technology infrastructure, 287-299, Springer, 2005.

Bernhardi, E. H., Cilliers, P. J., and Gaunt, C. T.: Improvement in the modelling of geomagnetically induced currents in southern Africa. S. Afr. J. Sci., 104, 265-272, 2008.

Blake, S. P., Gallagher, P. T., McCauley, J., Jones, A. G., Hogg, C., Campanya, J., Beggan, C. D., Thomson, A. W. P., Kelly, G. S., and Bell, D.: Geomagnetically induced currents in the Irish power network during geomagnetic storms Space Weather, 14, 1136-1154, https://doi.org/10.1002/2016SW001534, 2016.

Boteler, D. H. and Pirjola, R. J.: Modelling geomagnetically induced currents produced by realistic and uniform electric fields, IEEE T. Power Deliv., 13, 1303-1308, 1998.

Boteler, D. H. and Pirjola, R. J.: Comparison of methods for modelling geomagnetically induced currents, Ann. Geophys., 32, 1177-1187, https://doi.org/10.5194/angeo-32-1177-2014, 2014.

Boteler, D. H., Pirjola, R. J., and Nevanlinna, H.: The effects of geomagnetic disturbances on electrical systems at the Earth's surface, Adv. Space Res., 22, 17-27, https://doi.org/10.1016/S02731177(97)01096-X, 1998.

da Silva Barbosa, C., Hartmann, G. A., and Jasbinschek Pinheiro, K.: Numerical modeling of geomagnetically induced currents in 
a Brazilian transmission line, Adv. Space Res., 44, 1168-1179, https://doi.org/10.1016/j.asr.2014.11.008, 2015.

Duscher, K. and Günther, A.: Die Internationale Hydrogeologische Karte von Europa 1:1.500.000 (IHME1500), BGR, 2014.

Gaunt, C. T. and Coetzee, G.: Transformer failures in regions incorrectly considered to have low GIC-risk, Power Tech, 2007 IEEE Lausanne, 807-812, https://doi.org/10.1109/PCT.2007.4538419, 2007.

Halbedl, T., Renner, H., Sakulin, M., and Achleitner, G.: Measurement and analysis of neutral point currents in a 400-kv-network, Electric Power Quality and Supply Reliability Conference (PQ), 2014, 65-68, 2014.

Halbedl, T., Renner, H., Bailey, R. L., Leonhardt, R., and Achleitner, G.: Analysis of the impact of geomagnetic disturbances on the austrian transmission grid, Power Systems Computation Conference (PSCC), 1-5, 2016.

Heinson, G. S. and Lilley, F. E. M.: An application of thinsheet electromagnetic modelling to the Tasman Sea, Phys. Earth Planet. In., 81, 231-251, 1993.

Hejda, P. and Bochnícek, J.: Geomagnetically induced pipe-to-soil voltages in the Czech oil pipelines during October-November 2003, Ann. Geophys., 23, 3089-3093, https://doi.org/10.5194/angeo-23-3089-2005, 2005.

Horton, R., Boteler, D., Overbye, T. J., Pirjola, R., and Dugan, R. C.: A test case for the calculation of geomagnetically induced currents. IEEE T. Power Deliv., 27, 2368-2373, 2012.

Kappenman, J. G.: An overview of the impulsive geomagnetic field disturbances and power grid impacts associated with the violent Sun-Earth connection events of 29-31 October 2003 and a comparative evaluation with other contemporary storms, Space Weather, 3, S08C01, https://doi.org/10.1029/2004SW000128, 2005.

Lehtinen, M. and Pirjola, R.: Currents produced in earthed conductor networks by geomagnetically-induced electric fields. Ann. Geophys., 3, 479-484, 1985.

Marshall, R. A., Smith, E. A., Francis, M. J., Waters, C. L., and Sciffer, M. D.: A preliminary risk assessment of the Australian region power network to space weather, Space Weather, 9, S10004, https://doi.org/10.1029/2011SW000685, 2011.

Matandirotya, E., Cilliers, P. J., and Van Zyl, R. R.: Modeling geomagnetically induced currents in the South African power transmission network using the finite element method, Space Weather, 13, 185-195, https://doi.org/10.1002/2014SW001135, 2015.

Matsushita, S. and Maeda, H.: On the geomagnetic solar quiet daily variation field during the IGY, J. Geophys. Res., 70, 2535-2558, 1965.

Molinski, T. S.: Why utilities respect geomagnetically induced currents, J. Atmos. Sol.-Terr. Phy., 64, 1765-1778, 2002.

Myllys, M., Viljanen, A., Rui, Ø. A., and Ohnstad, T. M.: Geomagnetically induced currents in Norway: the northernmost highvoltage power grid in the world, J. Space Weather Space Clim., 4, A10, https://doi.org/10.1051/swsc/2014007, 2014.

Pirjola, R.: Fundamentals about the flow of geomagnetically induced currents in a power system applicable to estimating space weather risks and designing remedies, J. Atmos. Sol.-Terr. Phy., 64, 1967-1972, 2002.

Pirjola, R.: Study of effects of changes of earthing resistances on geomagnetically induced currents in an elec- tric power transmission system, Radio Sci., 43, RS1004, https://doi.org/10.1029/2007RS003704, 2008.

Pirjola, R., Viljanen, A., and Pulkkinen, A.: Space weather risk in power systems and pipelines, Phys. Chem. Earth Pt. C, 25, 333 337, https://doi.org/10.1016/S1464-1917(00)00027-1, 2000.

Price, A. T.: The induction of electric currents in non-uniform thin sheets and shells, Q. J. Mech. Appl. Math., 2, 283-310, 1949.

Price, P. R.: Geomagnetically induced current effects on transformers, IEEE T. Power Deliv., 17, 1002-1008, https://doi.org/10.1109/TPWRD.2002.803710, 2002.

Schubert, G.: Hydrogeologische Karte der Republik Österreich $1: 500$ 000, GBA/BMLFUW, Vienna, Austria, 2003.

Thomson, A. W. P., McKay, A. J., Clarke, E., and Reay, S. J.: Surface electric fields and geomagnetically induced currents in the Scottish Power grid during the 30 October 2003 geomagnetic storm, Space Weather, 3, S11002, https://doi.org/10.1029/2005SW000156, 2005.

Torta, J. M., Serrano, L., Regué, J. R., Sánchez, A. M., and Roldán, E.: Geomagnetically induced currents in a power grid of northeastern Spain, Space Weather, 10, S06002, https://doi.org/10.1029/2012SW000793, 2012.

Torta, M., Marsal, S., and Quintana, M.: Assessing the hazard from geomagnetically induced currents to the entire highvoltage power network in spain, Earth Planets Space, 66, 87, https://doi.org/10.1186/1880-5981-66-87, 2014.

Vasseur, G. and Weidelt, P.: Bimodal electromagnetic induction in non-uniform thin sheets with an application to the northern Pyrenean induction anomaly, Geophys. J. Int., 51, 669-690, 1977.

Viljanen, A. and Pirjola, R.: Geomagnetically induced currents in the Finnish high-voltage power system, Surv. Geophys., 15, 383408, 1994.

Viljanen, A.,Pirjola, R., Prácser, E., Katkalov, J., and Wik, M.: Geomagnetically induced currents in Europe, J. Space Weather Space Clim., 4, A09, https://doi.org/10.1051/swsc/2014006, 2014.

Wait, J. R.: Theory of magnetotelluric fields, J. Res. NBS D, 66, 509-541, 1962.

Watari, S., Kunitake, M., Kitamura, K., Hori, T., Kikuchi, T., Shiokawa, K., Nishitani, N., Kataoka, R., Kamide, Y., Aso, T., Watanabe, Y., and Tsuneta, Y.: Measurements of geomagnetically induced current in a power grid in Hokkaido, Japan, Space Weather, 7, S03002, https://doi.org/10.1029/2008SW000417, 2009.

Weaver, J. T.: Regional induction in Scotland: an example of threedimensional numerical modelling using the thin sheet approximation, Phys. Earth Planet. In., 28, 161-180, 1982.

Weckmann, U., Magunia, A., and Ritter, O.: Effective noise separation for magnetotelluric single site data processing using a frequency domain selection scheme, Geophys. J. Int., 161, 635-652, 2005.

Wik, M., Viljanen, A., Pirjola, R., Pulkkinen, A., Wintoft, P., and Lundstedt, H.: Calculation of geomagnetically induced currents in the $400 \mathrm{kV}$ power grid in southern Sweden, Space Weather, 6, 07005, https://doi.org/10.1029/2007SW000343, 2008.

Zois, I. P.: Solar activity and transformer failures in the Greek national electric grid, J. Space Weather Space Clim., 3, A32, https://doi.org/10.1051/swsc/2013055, 2013. 